typeset using JPSJ.sty $<$ ver.1.0b $>$

\title{
Energetics of Open Systems and Chemical Potential From Micro-Dynamics Viewpoints
}

\author{
Tatsuo Shibata* and Ken Sekimoto ${ }^{1, * *}$ \\ Research Institute for Mathematical Sciences, Kyoto University, Kyoto, 606-8502, Japan \\ ${ }^{1}$ Yukawa Institute for Theoretical Physics, Kyoto University, Kyoto 606-8502, Japan
}

(Received February 25, 2000 )

\begin{abstract}
We present the energetic aspect of open systems which may exchange particles with their environments. Our attention shall be paid to the scale that the motion of the particles is described by the classical Langevin dynamics. Along a particular realization of the stochastic process, we study the energy transfer into the open system from the environments. We are able to clarify how much energy each particle carries when it enters or leaves the system. On the other hand, the chemical potential should be considered as the concept in macro scale, which is relevant to the free energy potential with respect to the number of particles.
\end{abstract}

KEYWORDS: open systems, stochastic energetics, chemical potential

\section{§1. Introduction}

Open systems may exchange matter with their environments, in contact with a heat reservoir. The energy transfer into such a system from its environments may also take place. From the viewpoint of energetics, the question may arise as to how much energy the system gains when a particle enters or leaves the system. In particular, we may ask the question of how much energy a particle carries upon migration. For instance, we may imagine a macromolecule which can bind some small molecule, and may study the variation in the energy of the macromolecule upon binding. ${ }^{1)}$ According to the thermodynamic relation of open systems: $\Delta E=W+T \Delta S+\mu \Delta n$, one may expect that each particle which enters into the system carries the energy equal to the chemical potential $\mu$. However, we should notice that the thermodynamic relation only indicates the change in the thermodynamic state variables in a quasi-static process. In other words, we can evidently not discuss the energetic aspect of a particular event of particle exchange, based on the thermodynamic relation. Indeed, we will show that such an argument is not valid. In this Paper, the question will be approached from the viewpoint of the microscopic dynamics which describes the motion of particles entering or leaving the system. From such a viewpoint, we will study the energy balance of open systems upon exchanging a particle. We shall see that the chemical potential is obtained as the concepts in the macroscopic level that we find by considering the ensemble of the dynamics.

In order to study the energy transfer accompanied by the migration of the particles, we need a model of the system which can express the dynamics of particles ${ }^{2-4}$ ) and we need to formulate an energetic interpretation of the model. In the present Paper, first we suppose that a closed system in contact with a heat reservoir consists of $N$ particles. Then, we suppose a subsystem of the closed system. The subsystem can exchange the particles with the rest of the closed system. Thus, we can consider the subsystem as an open system. By studying the energy transfer of the open system, we construct the energetics associated with the particle exchange.

Let us first suppose a closed system denoted by $\Omega_{0}$ which consists of the $N$ particles. The dynamics of these particles is assumed to be described properly by the Langevin dynamics (here we only consider the overdamped case), given by

$$
\gamma \frac{\mathrm{d} x_{i}(t)}{\mathrm{d} t}=-\frac{\partial U(\boldsymbol{x}(t), a(t))}{\partial x_{i}}+\xi_{i}(t),
$$

where $x_{i}$ is the position of the $i$-th particle, $U(\boldsymbol{x}, a)$ is a potential energy with $\boldsymbol{x} \equiv\left(x_{1}, \cdots, x_{N}\right)$ and a controllable parameter $a, \gamma$ is a friction constant, and $\xi_{i}(t)$ is the white Gaussian processes which is characterized by $\left\langle\xi_{i}(t)\right\rangle=0$, and $\left\langle\xi_{i}\left(t_{1}\right) \xi_{j}\left(t_{2}\right)\right\rangle=2 \gamma \beta^{-1} \delta_{i, j} \delta\left(t_{1}-t_{2}\right)$ with $\beta^{-1}=k_{B} T$. $k_{B}$ is the Boltzmann constant and $T$ is temperature of the heat reservoir. Throughout this Paper, we consider the processes in which the parameter $a$ is supposed to be changed during the time interval from $t=0$ to $t=\tau$ by some external system according to a given protocol $a(t)$ from $a(0)=a_{i}$ to $a(\tau)=a_{f}$.

For the energetic interpretation of the Langevin dynamics, we adopt the stochastic energetics. ${ }^{5-7)}$ Let us consider both the friction and fluctuation terms in Eq.(1.1) as the force which the heat reservoir exerts on the system. Then, the work done on the system by the heat reservoir is given by

$$
\begin{aligned}
& \sum_{i=1}^{N} \int_{0}^{\tau}\left(-\gamma \frac{\mathrm{d} x_{i}(t)}{\mathrm{d} t}+\xi_{i}(t)\right) \mathrm{d} x_{i}(t) \\
& =\int_{0}^{\tau} \frac{\partial U(\boldsymbol{x}(t), a(t))}{\partial \boldsymbol{x}} \mathrm{d} \boldsymbol{x}(t)
\end{aligned}
$$



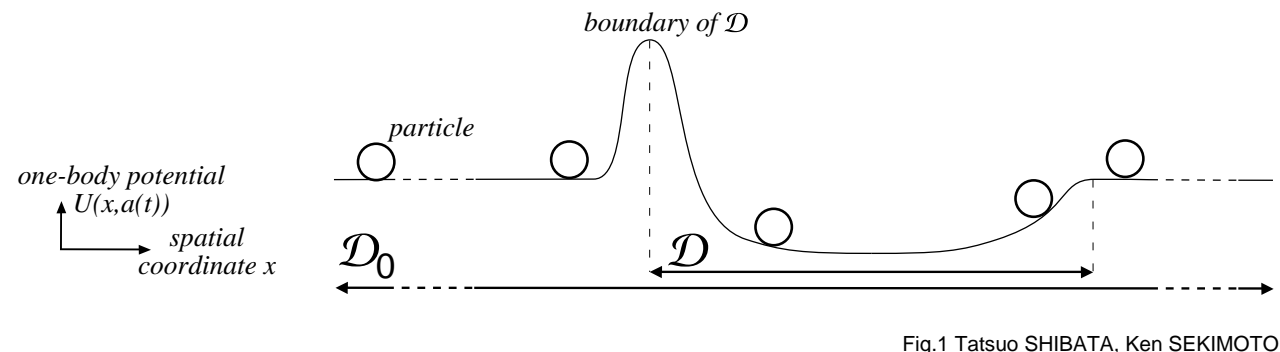

Fig. 1. Schematic diagram of the present situation. The open system $\Omega$ consists of the particles being in the spatial domain $\mathcal{D} \subset \mathcal{D}_{0}$. Hence, $\Omega$ may exchange particles with its surrounding. How much energy do the particles carry when these enter or leave $\Omega$ ?

$$
=\int_{0}^{\tau} \mathrm{d} U(t)-\int_{0}^{\tau} \frac{\partial U(\boldsymbol{x}(t), a(t))}{\partial a} \mathrm{~d} a(t),
$$

where we use Eq.(1.1) and $\mathrm{d} U=\frac{\partial U}{\partial a} \mathrm{~d} a+\frac{\partial U}{\partial \boldsymbol{x}} \mathrm{d} \boldsymbol{x}$. The left hand side defines the heat $Q\left[\left(\boldsymbol{x}(0), a_{i}\right) \rightarrow\left(\boldsymbol{x}(\tau), a_{f}\right)\right]$ along a particular trajectory. The notation in the bracket $[\cdot]$ indicates that the quantity takes a particular value which is obtained along a particular trajectory $\boldsymbol{x}(t)$ and the protocol $a(t)$. We should notice that the above stochastic integral is interpreted as the Stratonovich integral. $\left.{ }^{8}\right)$ Throughout this Paper, the stochastic integral is used in the Stratonovich sense.

The first term on the right hand side of Eq.(1.2) is the change in the internal energy $E(t)=U(\boldsymbol{x}(t), a(t))$, which we denote by $\Delta E\left[\left(\boldsymbol{x}(0), a_{i}\right) \rightarrow\left(\boldsymbol{x}(\tau), a_{f}\right)\right]$. The second term is interpreted as the work $W\left[\left(\boldsymbol{x}(0), a_{i}\right) \rightarrow\left(\boldsymbol{x}(\tau), a_{f}\right)\right]$ done on the system by some external system with changing the parameter $a(t)$ from $a(0)=a_{i}$ to $a(\tau)=a_{f}$. In this way, along one trajectory, the energy balance relation $^{5)}$ is obtained as

$$
\begin{aligned}
& \Delta E\left[\left(\boldsymbol{x}(0), a_{i}\right) \rightarrow\left(\boldsymbol{x}(\tau), a_{f}\right)\right] \\
& =W\left[\left(\boldsymbol{x}(0), a_{i}\right) \rightarrow\left(\boldsymbol{x}(\tau), a_{f}\right)\right] \\
& \quad+Q\left[\left(\boldsymbol{x}(0), a_{i}\right) \rightarrow\left(\boldsymbol{x}(\tau), a_{f}\right)\right] .
\end{aligned}
$$

This Paper has been organized as follows. A model of the open system will be introduced in Section 2. The energy balance of open systems along a particular trajectory is shown in Eq.(2.17). Then we find how much energy each particle carries at the boundary of the open system. In Section 3, it shall be shown that our study of open system is consistent with thermodynamics. It will be clarified that the chemical potential is relevant to the concept of free energy. Then the amount of work onto the open system in the quasi-static process is discussed and the thermodynamic relation is obtained. In Section 4, we will compare the energy balance relation with the thermodynamic relation in Eq.(4.1). Then, we conclude that the energy which a particle carries upon migration is not equal to the chemical potential $\mu$. We also show some remarks and future problems.

\section{§2. The Energy Balance Relation of Open Sys- tems}

Let us suppose that the particles of the closed system $\Omega_{0}$ are confined in a spatial domain $\mathcal{D}_{0}$, and the open system denoted by $\Omega$ consists of the particles being in the spatial domain $\mathcal{D} \subset \mathcal{D}_{0}$. Using some function $f(x)$, the spatial domain $\mathcal{D}$ is specified by $f\left(x_{i}\right) \leq c$. Hence, $\Omega$ may exchange particles with its surroundings. See Fig.1 for a schematic illustration of the situation.

We should note the possibility that no particle is in $\mathcal{D}$. In such a case, $\Omega$ contains no degrees of freedom. In order to avoid such a situation, it may be easy to introduce another degrees of freedom into $\Omega_{0}$, that is, a "transducer" which interacts with the particles being in $\mathcal{D}$. Then we can define $\Omega$ which consists of the transducer and the particles interacting with the transducer. However, it would not provide further physical insight. In order to present our idea simply, here we allow the situation that $\Omega$ has no degrees of freedom.

In the following Subsections, we study the energy balance relation of the open systems.

\subsection{Open systems as a subsystem of a closed system: One particle case}

Before considering many particle systems, we present the one particle system, i.e., $N=1$.

Using the Heviside function $\theta(x)$,

$$
\theta(x)=\left\{\begin{array}{ll}
1 & (x \geq 0) \\
0 & (x<0)
\end{array},\right.
$$

we define the internal energy $E(t)$ of $\Omega$ at time $t$, given by

$$
E(t)=\theta\left(c-f\left(x_{1}(t)\right)\right) U\left(x_{1}(t), a(t)\right)
$$

We suppose $U\left(x_{1}, a\right)$ depends on $a$ if $x_{1} \in \mathcal{D}$, i.e., $\mathrm{d} U\left(x_{1}, a\right) / \mathrm{d} a=0$ if $x_{1} \notin \mathcal{D}$. Then, the change in the internal energy during the process is given by

$$
\begin{aligned}
& \Delta E {\left[\left(x_{1}(0), a_{i}\right) \rightarrow\left(x_{1}(\tau), a_{f}\right)\right]=\int_{0}^{\tau} \mathrm{d} E(t) } \\
&=\int_{0}^{\tau} \theta\left(c-f\left(x_{1}(t)\right)\right) \frac{\partial U\left(x_{1}(t), a(t)\right)}{\partial a} \mathrm{~d} a(t)
\end{aligned}
$$




$$
\begin{aligned}
& +\int_{0}^{\tau} \theta\left(c-f\left(x_{1}(t)\right)\right) \frac{\partial U\left(x_{1}(t), a(t)\right)}{\partial x_{1}} \mathrm{~d} x_{i}(t) \\
& +\int_{0}^{\tau} \frac{\partial \theta\left(c-f\left(x_{1}(t)\right)\right)}{\partial x_{1}} U\left(x_{1}(t), a(t)\right) \mathrm{d} x_{1}(t)
\end{aligned}
$$

The first term on the right hand side is the mechanical work $W\left[\left(x_{1}(0), a_{i}\right) \rightarrow\left(x_{1}(\tau), a_{f}\right)\right]$ on $\Omega$ done by the external system. The second term is rewritten by substituting the Langevin equation Eq.(1.1) as

$$
\int_{0}^{\tau} \theta\left(c-f\left(x_{1}(t)\right)\right)\left(-\gamma \frac{\mathrm{d} x_{1}(t)}{\mathrm{d} t}+\xi_{1}(t)\right) \mathrm{d} x_{1}(t) .
$$

Hence, this term is the heat $Q\left[\left(x_{1}(0), a_{i}\right) \rightarrow\left(x_{1}(\tau), a_{f}\right)\right]$ transfered into $\Omega$ from the heat reservoir. The third term on the right hand side of Eq.(2.3) is transformed into

$$
\begin{aligned}
& -\int_{0}^{\tau} \frac{\mathrm{d} f\left(x_{1}\right)}{\mathrm{d} x_{1}} \delta\left(f\left(x_{1}\right)-c\right) U\left(x_{1}, a(t)\right) \mathrm{d} x_{1}(t) \\
& =-\int_{0}^{\tau} \frac{\mathrm{d} f\left(x_{1}(t)\right)}{\mathrm{d} t} \sum_{f\left(x_{1}\left(t^{\text {mig }}:\right)\right)=c} \frac{\delta\left(t-t^{\text {mig }}\right)}{\left|\frac{\mathrm{d} f\left(x_{1}\left(t^{\text {mig }}\right)\right)}{\mathrm{d} t}\right|} U\left(x_{1}(t), a(t)\right) \mathrm{d} t \\
& =\sum_{t=t^{\text {in }}} U\left(x_{1}(t), a(t)\right)-\sum_{t=t^{\text {out }}} U\left(x_{1}(t), a(t)\right)
\end{aligned}
$$

where $t^{\text {in }}$ and $t^{\text {out }}$ indicate all the instants that satisfy $f\left(x_{1}\left(t^{i n}\right)\right)=c, \mathrm{~d} f\left(x_{1}\left(t^{i n}\right)\right) / \mathrm{d} t<0$ and $f\left(x_{1}\left(t^{\text {out }}\right)\right)=$ $c, \mathrm{~d} f\left(x_{1}\left(t^{\text {out }}\right)\right) / \mathrm{d} t>0$ respectively. That is, $+U\left(x_{1}, a\right)$ is added when $f\left(x_{1}(t)\right)-c$ varies from positive to negative and $-U\left(x_{1}, a\right)$ is added when $f\left(x_{1}(t)\right)-c$ varies from negative to positive. Hence, the value of this term increase or decrease by $U\left(x_{1}, a\right)$ when the orbit runs across the boundary of $\mathcal{D}$ at $x_{1}$. We call this term $Q^{m i g}\left[\left(x_{1}(0), a_{i}\right) \rightarrow\left(x_{1}(\tau), a_{f}\right)\right]$.

Therefore, Eq.(2.3) is rewritten as

$$
\begin{aligned}
& \Delta E\left[\left(x_{1}(0), a_{i}\right) \rightarrow\left(x_{1}(\tau), a_{f}\right)\right] \\
& =W\left[\left(x_{1}(0), a_{i}\right) \rightarrow\left(x_{1}(\tau), a_{f}\right)\right] \\
& \quad+Q\left[\left(x_{1}(0), a_{i}\right) \rightarrow\left(x_{1}(\tau), a_{f}\right)\right] \\
& \quad+Q^{m i g}\left[\left(x_{1}(0), a_{i}\right) \rightarrow\left(x_{1}(\tau), a_{f}\right)\right],
\end{aligned}
$$

which displays the energy balance relation of the system.

\subsection{Many particle systems with interactions}

Let us next study the many particle systems, i.e., the closed system $\Omega_{0}$ contains $N$ interacting particles.

The interaction potential of the particles is the sum of a $n$-body potential for $n=1,2, \cdots$, given by,

$$
U(\boldsymbol{x}, a)=\sum_{i_{1}=1}^{N}\left(\phi_{i_{1}}^{(1)}(a)+\sum_{i_{2}=i_{1}+1}^{N}\left(\phi_{i_{1}, i_{2}}^{(2)}+\cdots\right)\right)
$$

where $\phi_{i_{1}, \cdots, i_{n}}^{(n)} \equiv \phi^{(n)}\left(x_{i_{1}}, \cdots, x_{i_{n}}\right)$ is the $n$-body energy potential of $x_{i_{1}}, \cdots, x_{i_{n}}$. We assume that the one body energy potential $\phi_{i}^{(1)}(a) \equiv \phi^{(1)}\left(x_{i}, a\right)$ depends on $a$ if $x_{i} \in \mathcal{D}$, i.e., $d \phi^{(1)}(a) / \mathrm{d} a=0$ if $x_{i} \notin \mathcal{D}$. We assume only short range interactions so that the $n$ body potential has non-zero value only when all the $n$ particles are close to each other.

As in the case of the one particle system, the internal energy $E$ of $\Omega$ is defined by considering whether each particle is in $\mathcal{D}$ or not:

$$
\begin{aligned}
E= & \sum_{i_{1}=1}^{N}\left(\theta_{i_{1}}^{(1)} \phi_{i_{1}}^{(1)}(a)+\sum_{i_{2}=i_{1}+1}^{N}\left(\theta_{i_{1}, i_{2}}^{(2)} \phi_{i_{1}, i_{2}}^{(2)}\right.\right. \\
& \left.\left.+\sum_{i_{3}=i_{2}+1}^{N}\left(\theta_{i_{1}, i_{2}, i_{3}}^{(3)} \phi_{i_{1}, i_{2}, i_{3}}^{(3)}+\cdots\right)\right)\right),
\end{aligned}
$$

where $\theta_{i_{1}, \cdots, i_{n}}^{(n)}$ is a function indicating whether the $n$ particles belong to $\Omega$ or not. Using the Heviside function $\theta(x), \theta_{i_{1}, \cdots, i_{n}}^{(n)} \equiv \theta^{(n)}\left(x_{i_{1}}, \cdots, x_{i_{n}}\right)$ is defined by

$$
\begin{aligned}
\theta_{i_{1}}^{(1)} & \equiv \theta\left(c-f\left(x_{i_{1}}\right)\right), \\
\theta_{i_{1}, i_{2}}^{(2)} & \equiv 1-\left(1-\theta_{i_{1}}^{(1)}\right)\left(1-\theta_{i_{2}}^{(1)}\right), \\
\theta_{i_{1}, \cdots, i_{n}}^{(n)} & \equiv 1-\left(1-\theta_{i_{1}}^{(1)}\right)\left(1-\theta_{i_{2}}^{(1)}\right) \cdots\left(1-\theta_{i_{n}}^{(1)}\right) .
\end{aligned}
$$

The value of $\theta_{i_{1}, \cdots, i_{n}}^{(n)}$ is unity when at least one of the $n$ particles is in $\Omega$, or is zero, otherwise. This means that certain $n$ particles belong to $\Omega$ when at least one particle of these particles is inside $\mathcal{D}$. Thus, $\theta_{i_{1}, \cdots, i_{n}}^{(n)}=1$ and the interaction energy $\phi_{i_{1}, \cdots, i_{n}}^{(n)}$ of the $n$ particles is included in the internal energy $E$. However, if $m(<n)$ particles $j_{1}, \cdots, j_{m}$ of these $n$ particles are outside $\mathcal{D}$, $\theta_{j_{1}, \cdots, j_{m}}^{(m)}=0$ and the interaction energy $\phi_{j_{1}, \cdots, j_{m}}^{(m)}$ of these $m$ particles is excluded from the internal energy $E$.

The change in the internal energy along a particular trajectory is given by,

$$
\begin{aligned}
& \Delta E\left[\left(\boldsymbol{x}(0), a_{i}\right) \rightarrow\left(\boldsymbol{x}(\tau), a_{f}\right)\right] \\
& =\sum_{i=1}^{N} \int_{0}^{\tau} \theta_{i}^{(1)}(t) \frac{\partial \phi_{i}^{(1)}(a(t))}{\partial a} \mathrm{~d} a(t) \\
& \quad+\sum_{i=1}^{N} \int_{0}^{\tau} \theta_{i}^{(1)}(t)\left(-\gamma \frac{\mathrm{d} x_{i}(t)}{\mathrm{d} t}+\xi_{i}(t)\right) \mathrm{d} x_{i}(t) \\
& \quad+\sum_{i=1}^{N} \int_{0}^{\tau} \frac{\partial \theta_{i}^{(1)}(t)}{\partial x_{i}} \phi_{i}(t) \mathrm{d} x_{i}(t) \\
& \quad+\sum_{i=1}^{N} \int_{0}^{\tau} \frac{\partial \phi_{i}^{\partial \mathcal{D}}(t)}{\partial x_{i}} \mathrm{~d} x_{i}(t)
\end{aligned}
$$

with

$$
\begin{gathered}
\phi_{i}=\phi_{i}^{(1)}+\sum_{\substack{j_{1}=1 \\
j_{1} \neq i}}^{N}\left(\phi_{i, j_{1}}^{(2)}+\sum_{\substack{j_{2}=j_{1}+1 \\
j_{2} \neq i}}^{N}\left(\phi_{i, j_{1}, j_{2}}^{(3)}+\cdots\right.\right. \\
\left.\left.\cdots+\sum_{\substack{j_{N-1}=j_{N-2}+1 \\
j_{N-1} \neq i}}^{N} \phi_{i, j_{1}, \cdots, j_{N-1}}^{(N)}\right)\right),
\end{gathered}
$$

and

$$
\begin{gathered}
\phi_{i}^{\partial \mathcal{D}}=\left(1-\theta_{i}^{(1)}\right) \sum_{\substack{j_{1}=1 \\
j_{1} \neq i}}^{N}\left(\theta_{j_{1}}^{(1)} \phi_{i, j_{1}}^{(2)}+\sum_{\substack{j_{2}=j_{1}+1 \\
j_{2} \neq i}}^{N}\left(\theta_{j_{1}, j_{2}}^{(2)} \phi_{i, j_{1}, j_{2}}^{(3)}+\cdots\right.\right. \\
\left.\left.\cdots+\sum_{\substack{j_{N-1}=j_{N-2}+1 \\
j_{N-1} \neq i}}^{N} \theta_{j_{1}, \cdots, j_{N-1}}^{(N-1)} \phi_{i, j_{1}, \cdots, j_{N-1}}^{(N)}\right)\right) .
\end{gathered}
$$


Eq.(2.10) is obtained by the following transformation. The total derivative of the internal energy Eq.(2.8) is written as

$$
\begin{aligned}
\mathrm{d} E= & \sum_{i=1}^{N} \theta_{i}^{(1)} \frac{\partial \phi_{i}(a)}{\partial a} \mathrm{~d} a \\
& +\sum_{i=1}^{N} \frac{\partial}{\partial x_{i}}\left(\sum _ { j _ { 1 } = 1 } ^ { N } \left(\theta_{j_{1}}^{(1)} \phi_{j_{1}}^{(1)}+\sum_{j_{2}=j_{1}+1}^{N}\left(\theta_{j_{1}, j_{2}}^{(2)} \phi_{j_{1}, j_{2}}^{(2)}+\cdots\right.\right.\right. \\
& \left.\left.\left.\cdots+\sum_{j_{N}=j_{N-1}+1}^{N} \theta_{j_{1}, \cdots, j_{N}}^{(N)} \phi_{j_{1}, \cdots, j_{N}}^{(N)}\right)\right)\right) \mathrm{d} x_{i}
\end{aligned}
$$

Considering that $\theta_{i_{1}, \cdots, i_{N}}^{(N)}$ and $\phi_{i_{1}, \cdots, i_{N}}^{(N)}$ are symmetric functions of $x_{i_{1}}, \cdots, x_{i_{N}}$, the second term on the right hand side of Eq.(2.13) is rewritten as

$$
\begin{aligned}
& \sum_{i=1}^{N} \frac{\partial}{\partial x_{i}}\left(\theta_{i}^{(1)} \phi_{i}^{(1)}+\sum_{\substack{j_{1}=1 \\
j_{1} \neq i}}^{N}\left(\theta_{i, j_{1}}^{(2)} \phi_{i, j_{1}}^{(2)}+\sum_{\substack{j_{2}=j_{1}+1 \\
j_{2} \neq i}}^{N}\left(\theta_{i, j_{1}, j_{2}}^{(3)} \phi_{i, j_{1}, j_{2}}^{(3)}+\cdots\right.\right.\right. \\
& \left.\left.\left.\cdots+\sum_{\substack{j_{N-1}=j_{N-2}+1 \\
j_{N-1} \neq i}}^{N} \theta_{i, j_{1}, \cdots, j_{N-1}}^{(N)} \phi_{i, j_{1}, \cdots, j_{N-1}}^{(N)}\right)\right)\right) \mathrm{d} x_{i}
\end{aligned}
$$

Noting that $\theta_{i_{1}, \cdots, i_{n}}^{(n)}$ is transformed as $\theta_{i_{1}, \cdots, i_{n}}^{(n)}=\theta_{i_{1}}^{(1)}+$ $\left(1-\theta_{i_{1}}^{(1)}\right) \theta_{i_{2}, \cdots, i_{n}}^{(n-1)}$, then Eq.(2.14) is rewritten as

$$
\sum_{i=1}^{N}\left(\theta_{i}^{(1)} \frac{\partial \phi_{i}}{\partial x_{i}}+\frac{\partial \theta_{i}^{(1)}}{\partial x_{i}} \phi_{i}+\frac{\partial \phi_{i}^{\partial \mathcal{D}}}{\partial x_{i}}\right) \mathrm{d} x_{i}
$$

with Eqs.(2.11) and (2.12). Using the relation $\frac{\partial \phi_{i}}{\partial x_{i}}=$ $-\gamma \frac{\mathrm{d} x_{i}}{\mathrm{~d} t}+\xi_{i}$, Eq.(2.15) is rewritten as

$$
\sum_{i=1}^{N}\left(\theta_{i}^{(1)}\left(-\gamma \frac{\mathrm{d} x_{i}}{\mathrm{~d} t}+\xi_{i}\right)+\frac{\partial \theta_{i}^{(1)}}{\partial x_{i}} \phi_{i}+\frac{\partial \phi_{i}^{\partial \mathcal{D}}}{\partial x_{i}}\right) \mathrm{d} x_{i} .
$$

Substituting Eq.(2.16) into Eq.(2.13), and integrating it with respect to $\boldsymbol{x}(t)$ and $a(t)$ from $t=0$ to $t=\tau$, then a simple calculation leads to Eq.(2.10).

The first term on the right hand side of Eq.(2.10) is the work $W\left[\left(\boldsymbol{x}(0), a_{i}\right) \rightarrow\left(\boldsymbol{x}(\tau), a_{f}\right)\right]$ on $\Omega$ done by the external system. The second term of Eq.(2.10) is the heat transfered from the heat reservoir to the particles being in $\mathcal{D}$, denote by $Q\left[\left(\boldsymbol{x}(0), a_{i}\right) \rightarrow\left(\boldsymbol{x}(\tau), a_{f}\right)\right]$. As is discussed in the previous Section, the third term of Eq.(2.10) equals to $\phi_{i}\left(t^{i n}\right)$ when the orbit $x_{i}(t)$ enters into $\mathcal{D}$ over the boundary at $t=t^{\text {in }}$, or equals to $-\phi_{i}\left(t^{\text {out }}\right)$ when the orbit $x_{i}(t)$ goes out of $\mathcal{D}$ at $t=t^{\text {out }}$. Let us denote this energy transfer accompanied by the migration of the particles by $Q^{m i g}\left[\left(\boldsymbol{x}(0), a_{i}\right) \rightarrow\left(\boldsymbol{x}(\tau), a_{f}\right)\right]$. The fourth term of Eq.(2.10) distinguishes the interacting particles systems from one particle systems or free particles systems. This term shows the contribution by the particles outside $\mathcal{D}$ which interacts with the particles inside $\mathcal{D}$ across the boundary $\partial \mathcal{D}$ of $\mathcal{D}$. Here, we denote this term as $Q^{\partial \mathcal{D}}\left[\left(\boldsymbol{x}(0), a_{i}\right) \rightarrow\left(\boldsymbol{x}(\tau), a_{f}\right)\right]$.

Therefore, Eq.(2.10) is rewritten as

$$
\begin{aligned}
& \Delta E\left[\left(\boldsymbol{x}(0), a_{i}\right) \rightarrow\left(\boldsymbol{x}(\tau), a_{f}\right)\right] \\
& \quad=W\left[\left(\boldsymbol{x}(0), a_{i}\right) \rightarrow\left(\boldsymbol{x}(\tau), a_{f}\right)\right]
\end{aligned}
$$

$$
\begin{aligned}
& +Q\left[\left(\boldsymbol{x}(0), a_{i}\right) \rightarrow\left(\boldsymbol{x}(\tau), a_{f}\right)\right] \\
& +Q^{m i g}\left[\left(\boldsymbol{x}(0), a_{i}\right) \rightarrow\left(\boldsymbol{x}(\tau), a_{f}\right)\right] \\
& +Q^{\partial \mathcal{D}}\left[\left(\boldsymbol{x}(0), a_{i}\right) \rightarrow\left(\boldsymbol{x}(\tau), a_{f}\right)\right]
\end{aligned}
$$

In this way, along a particular trajectory, we have the energy balance relation of the open systems with many body interactions.

In the similar way, the change in the ensemble average of the internal energy $E(t)$ can be also discussed. The ensemble average of the internal energy is defined by

$$
\langle E(t)\rangle=\int E(t) P(\boldsymbol{x}, t) \mathrm{d} \boldsymbol{x},
$$

where $P(\boldsymbol{x}, t)$ is the probability distribution function of the state of $\Omega_{0}$ obtained by solving the Fokker-Plank equation, and $\langle\cdot\rangle$ indicates the ensemble average over all the possible trajectory.

The energy balance relation of the mean values is

$$
\begin{aligned}
\Delta\left\langle E\left[a_{i} \rightarrow a_{f}\right]\right\rangle=\left\langle W\left[a_{i} \rightarrow a_{f}\right]\right\rangle+\left\langle Q\left[a_{i} \rightarrow a_{f}\right]\right\rangle \\
+\left\langle Q^{m i g}\left[a_{i} \rightarrow a_{f}\right]\right\rangle+\left\langle Q^{\partial \mathcal{D}}\left[a_{i} \rightarrow a_{f}\right]\right\rangle .
\end{aligned}
$$

Henceforth we use the notation $\left[a_{i} \rightarrow a_{f}\right]$ for those quantities concerning the processes in which $a(t)$ is changed from $a_{i} \rightarrow a_{f}$. Each term on the right hand side corresponds to each term in Eq.(2.17). We do not need to postulate further condition in order to obtain this expression for the mean values. Comparing Eq.(2.17) with the thermodynamic relation that we have presented in Section 1 , we have

$$
\langle Q\rangle+\left\langle Q^{m i g}\right\rangle+\left\langle Q^{\partial \mathcal{D}}\right\rangle=T \Delta S+\mu \Delta n,
$$

where we suppose the quasi-static process. We will discuss the meaning of this expression in Section 4.

Before concluding this Section, let us evaluate $Q^{\text {mig }}$ for the case that we are able to effectively exclude the possibility of the direct interaction across the boundary $\partial \mathcal{D}$, i.e. $Q^{\partial \mathcal{D}}=0 .{ }^{9)}$ We further suppose $U(\boldsymbol{x}, a)=$ $\tilde{U}(\boldsymbol{x}, a)$ defined by

$$
\tilde{U}(\boldsymbol{x}, a) \equiv \tilde{U}^{(n)}\left(x_{1}, \cdots, x_{n}, a\right)+(N-n) U_{0},
$$

when $x_{i}$ satisfies $f\left(x_{i}\right)<c,(i=1, \cdots, n)$ and $x_{j}$ satisfies $f\left(x_{j}\right) \geq c,(j=n+1, \cdots, N)$. This assumption leads to the simple form of $Q^{\text {mig }}$ as

$$
Q^{m i g}=U_{0}\left(\sum_{t=t^{\text {in }}} 1-\sum_{t=t^{\text {out }}} 1\right) .
$$

Even in the present simple case, the relation between the terms on the left and the right hand side of Eq.(2.20) does not seem evident. However, this does not imply that our study of the open system based on the stochastic energetics is incompatible with thermodynamics. In the next Section, we will show the consistency. Then in Section 4, Eq.(2.20) is consistently interpreted mentioning the difference of the scale of description on each term.

\section{§3. The thermodynamic relation from micro- scopic dynamics}

It has been shown in the previous works ${ }^{5-7}$ ) that the framework of the stochastic energetics is consistent with 
thermodynamics of the system in contact with a heat reservoir. In this Section, we shall show that even in the open system the stochastic energetics is consistent with thermodynamics. ${ }^{10)}$ First, in Subsection 3.1 we study the probability that the open system $\Omega$ is found to be a particular physical state which is specified by the number of particles in $\mathcal{D}$ with their positions. Then, we will find that the chemical potential is relevant to the concept of free energy potential obtained through the reduction of the description of the system. In Subsection 3.2, we also study the quasi-static process, in which the work onto the open system is shown to equal the difference of a pertinent thermodynamic potential. Then, in Subsection 3.3 the thermodynamic relation is obtained so that the compatibility of our study with thermodynamics shall be shown.

In the present Section, the state of the closed system $\Omega_{0}$ is specified by $\boldsymbol{x}^{(N)}=\left(x_{1}, \cdots, x_{N}\right)$ which constitute the phase space $\Gamma^{(N)}$. We assume that all the particles are identical. If two points in $\Gamma^{(N)}$ differ only by the permutation of $x_{i}$ 's they may be considered as representing the same physical state. Then, we can introduce the phase space $\Gamma^{\prime(N)}$ obtained by the identification of all such points. In $\Gamma^{\prime(N)}$ the state of the particles is specified by $\boldsymbol{x}^{(N)}$. For each point $\boldsymbol{x}^{\prime(N)}$ in $\Gamma^{\prime(N)}$, there are $N$ ! points in $\Gamma^{(N)}$. Hence, if $f\left(\boldsymbol{x}^{(N)}\right)$ is a symmetric function of all the $x_{i}$ 's then

$$
\int_{\left(\mathcal{D}_{0}\right)^{\prime}} f\left(\boldsymbol{x}^{\prime(N)}\right) \mathrm{d} \boldsymbol{x}^{\prime(N)}=\frac{1}{N !} \int_{\mathcal{D}_{0} N} f\left(\boldsymbol{x}^{(N)}\right) \mathrm{d} \boldsymbol{x}^{(N)},
$$

where $\left(\mathcal{D}_{0}{ }^{N}\right)^{\prime}$ indicates the domain in $\Gamma^{\prime(N)}$ which corresponds to $\mathcal{D}_{0}{ }^{N}$ in $\Gamma^{(N)}$.

\subsection{The chemical potential as free energy potential}

The equilibrium distribution function of $\boldsymbol{x}^{(N)}$ of $\Omega_{0}$ is obtained by solving the Fokker-Plank equation,

$$
P_{e q}\left(\boldsymbol{x}^{(N)}, a\right)=\frac{1}{\mathcal{Z}(a)} \frac{1}{v_{0}^{N}} e^{-\beta U\left(\boldsymbol{x}^{(N)}, a\right)} .
$$

with the normalization condition:

$$
1=\frac{1}{v_{0}^{N}} \frac{1}{\mathcal{Z}(a)} \int_{\left(\mathcal{D}_{0} N\right)} e^{-\beta U\left(\boldsymbol{x}^{(N)}, a\right)} \mathrm{d} \boldsymbol{x}^{(N)} .
$$

Here the constant $v_{0}$ of the dimension of volume has been introduced so that $\mathcal{Z}(a)$ is dimensionless. Since $U\left(\boldsymbol{x}^{(N)}, a\right)$ is symmetric function of $x_{i}$ 's, the probability distribution function of the state $\boldsymbol{x}^{\prime(N)}$ is

$$
P_{e q}^{\prime}\left(\boldsymbol{x}^{\prime(N)}, a\right)=\frac{N !}{\mathcal{Z}(a) v_{0}^{N}} e^{-\beta U\left(\boldsymbol{x}^{\prime(N)}, a\right)},
$$

with $\boldsymbol{x}^{\prime(N)} \in\left(\mathcal{D}_{0}^{N}\right)^{\prime}$.

By integrating $P_{e q}^{\prime}\left(\boldsymbol{x}^{\prime(N)}, a\right)$ with respect to the degrees of freedom of the particles being outside $\mathcal{D}$, that is, in $\mathcal{D}_{0} \backslash \mathcal{D}$, we obtain the probability distribution function of $\Omega$ that there are $n$ particles in $\mathcal{D}$ with their positions $\boldsymbol{x}^{\prime(n)} \in\left(\mathcal{D}^{n}\right)^{\prime}$ as

$$
P_{e q}^{\prime}\left(\boldsymbol{x}^{\prime(n)}, a\right)=\frac{1}{\mathcal{Z}(a)} \frac{1}{v_{0}^{n}} e^{-\beta \mathcal{U}^{(n)}\left(\boldsymbol{x}^{\prime(n)}, a\right)},
$$

where $\mathcal{U}^{(n)}\left(\boldsymbol{x}^{\prime(n)}\right)$ is considered as the free energy potential under the restriction that the state of $\Omega$ is $\boldsymbol{x}^{\prime(n)}$.
Hence, $\mathcal{U}^{(n)}\left(\boldsymbol{x}^{\prime(n)}\right)$ is given by

$$
e^{-\beta \mathcal{U}^{(n)}\left(\boldsymbol{x}^{\prime(n)}, a\right)}=\frac{N !}{v_{0}^{N-n}} \int_{\left(\left(\mathcal{D}_{0} \backslash \mathcal{D}\right)^{N-n}\right)^{\prime}} e^{-\beta U\left(\boldsymbol{x}^{\prime(N)}, a\right)} \mathrm{d} \boldsymbol{x}^{(N-n)},
$$

with $\boldsymbol{x}^{\prime(n)} \in\left(\mathcal{D}^{n}\right)^{\prime}$. We can show the following normalization condition holds:

$$
1=\sum_{n=0}^{N} \int_{\left(\mathcal{D}^{n}\right)^{\prime}} P_{e q}^{\prime(n)}\left(\boldsymbol{x}^{\prime(n)}, a\right) \mathrm{d} \boldsymbol{x}^{\prime(n)} .
$$

Hereafter we shall exclude the possibility of the direct interaction among the particles across the boundary $\partial \mathcal{D}$ and assume $U\left(\boldsymbol{x}^{(N)}, a\right)=\tilde{U}\left(\boldsymbol{x}^{(N)}, a\right)$, with Eq.(2.21). Then, Eq.(3.6) is rewritten as

$$
\begin{aligned}
& e^{-\beta \mathcal{U}^{(n)}\left(\boldsymbol{x}^{\prime(n)}, a\right)} \\
& =\frac{N !\left(V_{0}-V\right)^{N-n}}{v_{0}^{N-n}(N-n) !} e^{-\beta\left(\tilde{U}^{(n)}\left(\boldsymbol{x}^{\prime(n)}, a\right)+(N-n) U_{0}\right)},
\end{aligned}
$$

with $\boldsymbol{x}^{\prime(n)} \in\left(\mathcal{D}^{n}\right)^{\prime}$. We shall consider the case that the volume of $\Omega_{0}, V_{0} \equiv \int_{\mathcal{D}_{0}} 1 \mathrm{~d} x$ and $N$ are sufficiently large with $\rho \equiv N / V_{0}$ and $V \equiv \int_{\mathcal{D}} 1 \mathrm{~d} x$ being kept to be finite constants. Then, $\mathcal{U}^{(n)}\left(\boldsymbol{x}^{(n)}, a\right)$ is given by

$$
\begin{aligned}
& \mathcal{U}^{(n)}\left(\boldsymbol{x}_{n}, a\right)=\tilde{U}^{(n)}\left(\boldsymbol{x}^{(n)}, a\right)-n\left(U_{0}+\beta^{-1} \log \rho v_{0}\right) \\
& +N\left(U_{0}+\beta^{-1} \log \frac{v_{0}}{V_{0}}\right)+\beta^{-1} \rho V+\mathcal{O}\left(\frac{1}{N}\right) .
\end{aligned}
$$

Since, on the right hand side, only the first and the second terms depend on $\boldsymbol{x}^{\prime(n)}$ and on $n$, these terms should be regarded as a new free energy potential assigned to the situation where there are $n$ particles in $\mathcal{D}$ with their positions corresponding to the physical state $\boldsymbol{x}^{\prime(n)} \in\left(\mathcal{D}^{n}\right)^{\prime}$. Hence, introducing the chemical potential $\mu$ through the definition:

$$
\mu \equiv U_{0}+\beta^{-1} \log \rho v_{0},
$$

$P_{e q}^{\prime(n)}\left(\boldsymbol{x}^{\prime(n)}, a\right)$ is rewritten as

$$
P_{e q}^{\prime(n)}\left(\boldsymbol{x}^{\prime(n)}, a\right)=\frac{1}{\Xi(a)} \frac{1}{v_{0}^{n}} e^{-\beta\left(\tilde{U}^{(n)}\left(\boldsymbol{x}^{\prime(n)}, a\right)-\mu n\right)},
$$

where $\Xi(a)$ is a normalization constant given by

$$
\begin{aligned}
\Xi(a) & =\lim _{N \rightarrow \infty} \mathcal{Z}(a) e^{\beta N\left(U_{0}+\beta^{-1} \log \frac{v_{0}}{V_{0}}+\mathcal{O}\left(\frac{1}{N}\right)\right)} \\
& =\sum_{n=0}^{\infty} \frac{1}{v_{0}^{n}} \int_{\left(\mathcal{D}^{n}\right)^{\prime}} e^{-\beta\left(\tilde{U}^{(n)}\left(\boldsymbol{x}^{\prime(n)}, a\right)-\mu n\right)} \mathrm{d} \boldsymbol{x}^{\prime(n)}
\end{aligned}
$$

Eq.(3.11) is the grand canonical distribution.

Notice that the chemical potential $\mu$ is obtained as a part of the free energy potential $\mathcal{U}^{(n)}\left(x_{1}, \cdots, x_{n}, a\right)$. Hence, the crucial point for obtaining the chemical potential is to consider the trajectory $\boldsymbol{x}^{(N)}$ in the reduced space $\Gamma^{\prime(N)}$ rather than $\Gamma^{(N)}$ and to extract the effective potential of the state $\boldsymbol{x}^{\prime(n)} \in\left(\mathcal{D}^{n}\right)^{\prime}$ by eliminating the degrees of freedom in $\left(\left(\mathcal{D}_{0} \backslash \mathcal{D}\right)^{N-n}\right)^{\prime}$.

\subsection{Work on the open system in quasi-static process}

As is discussed in the previous Section, the work onto the open systems is a random variable, whose value is determined depending on a particular trajectory. If the process is quasi-static, however, we expect that the 
amount of work is deterministic quantity. In this Subsection, we show that the work onto the open system is given by the difference of a pertinent thermodynamic potential.

Here we note that the Langevin dynamics Eq.(1.1) has the ergodic property that long time averages can be replaced by the ensemble averages. When the change of the parameter $a$ is slow enough and hence $|\mathrm{d} a(t) / \mathrm{d} t|$ is small enough, the work along a particular trajectory given by $\int_{0}^{\tau} \frac{\partial U\left(\boldsymbol{x}^{\prime(N)}(t), a(t)\right)}{\partial a} \mathrm{~d} a(t)$ can be evaluated asymptotically as the integral of the equilibrium ensemble average of the integrand with respect to $a$ from $a_{i}$ to $a_{f}$. Using $P_{e q}^{\prime(n)}\left(\boldsymbol{x}^{\prime(n)}, a\right)$, the work done by the external system is written in the limit $\tau \rightarrow \infty$ as

$$
\begin{aligned}
& \lim _{\tau \rightarrow \infty} W\left[\left(\boldsymbol{x}(0), a_{i}\right) \rightarrow\left(\boldsymbol{x}(\tau), a_{f}\right)\right] \\
& =\int_{a_{i}}^{a_{f}} \mathrm{~d} a \sum_{n=0}^{N} \int_{\left(\mathcal{D}^{n}\right)^{\prime}} \mathrm{d} \boldsymbol{x}^{\prime(n)} \frac{\partial \tilde{U}\left(\boldsymbol{x}^{\prime(n)}, a(t)\right)}{\partial a} P_{e q}^{\prime(n)}\left(\boldsymbol{x}^{(n)}, a\right) \\
& =\Delta J\left[a_{i} \rightarrow a_{f}\right]=J\left(a_{f}\right)-J\left(a_{i}\right),
\end{aligned}
$$

where $J(a)$ is the grand canonical potential defined by $J(a)=-\beta^{-1} \log \Xi(a)$.

Hence, if the change of the parameter is slow enough, the work done by the external system onto the open system equals to the difference of the grand canonical potential with the probability being unity, without mentioning the ensemble average.

It should be noted that for a finite time interval, the amount of the work of a particular process can be larger or smaller than $\Delta J$. Considering the ensemble average of the work, the minimum work principle holds on the open system, ${ }^{10)}$ i.e., $\left\langle W\left[a_{i} \rightarrow a_{f}\right]\right\rangle \geq \Delta J\left[a_{i} \rightarrow a_{f}\right]$.

\subsection{Thermodynamic relation}

The equilibrium ensemble average of the internal energy of $\Omega$ is given by

$$
\begin{aligned}
&\langle E(a)\rangle= \sum_{n=0}^{N} \int_{\left(\mathcal{D}^{n}\right)^{\prime}} \tilde{U}^{(n)}\left(\boldsymbol{x}^{\prime(n)}\right) P_{e q}^{\prime(n)}\left(\boldsymbol{x}^{\prime(n)}, a\right) \mathrm{d} \boldsymbol{x}^{\prime(n)} \\
&=\sum_{n=0}^{N} \int_{\left(\mathcal{D}^{n}\right)^{\prime}}\left(-\beta^{-1} \log \left(v_{0}^{n} P_{e q}^{\prime(n)}\left(\boldsymbol{x}^{\prime(n)}, a\right)\right)+\mu n+J(a)\right) \\
& \quad \times P_{e q}^{\prime(n)}\left(\boldsymbol{x}^{\prime(n)}, a\right) \mathrm{d} \boldsymbol{x}^{\prime(n)},
\end{aligned}
$$

where we substitute Eq.(3.11) into $\tilde{U}^{(n)}\left(\boldsymbol{x}^{\prime(n)}, a\right)$. Introducing the entropy $S(a)$ given by

$$
S(a)=-k_{B} \sum_{n=0}^{N} \int_{\left(\mathcal{D}^{n}\right)^{\prime}} P_{e q}^{\prime(n)} \log \left(v_{0}^{n} P_{e q}^{\prime(n)}\right) \mathrm{d} \boldsymbol{x}^{\prime(n)},
$$

we have

$$
\langle E(a)\rangle=J(a)+T S(a)+\mu\langle n(a)\rangle,
$$

where $\langle n(a)\rangle$ is the equilibrium ensemble average of the number of particles in $\mathcal{D}$.

When the parameter $a$ is changed from $a_{i}$ to $a_{f}$ quasistatically, the change in the internal energy is given by,

$$
\Delta\left\langle E\left[a_{i} \rightarrow a_{f}\right]\right\rangle=W\left[a_{i} \rightarrow a_{f}\right]
$$

$$
+T \Delta S\left[a_{i} \rightarrow a_{f}\right]+\mu \Delta\left\langle n\left[a_{i} \rightarrow a_{f}\right]\right\rangle,
$$

where Eq.(3.13) is substituted. In this way, we have the thermodynamic relation of the open system, which holds on the ensemble of the trajectories $\boldsymbol{x}(t)$.

Eq.(3.17) may suggest that the increase in number of particles during a quasi-static process would accompany the transfer of energy into the system $\Omega$ as if each particle carries the chemical potential. In the next Section, we conclude this Paper, mentioning how the thermodynamic relation is compatible with the energy balance relation that we have obtained in Eq.(2.17).

\section{§4. The energy carried by a particle upon mi- gration: Conclusion}

In the preceding sections, we have constructed the energy balance relation Eq.(2.17) of the open system focusing our attention to the scale that the system is described by the Langevin dynamics. On the other hand, it has been clarified that the chemical potential, which reflects the probability that the open system contains a particular number of particles, is relevant to the free energy potential. Here, we shall illustrate the compatibility of the energy balance relation Eq.(2.17) with the thermodynamic relation Eq.(3.17), noting the difference of the scale of their description.

As is the case in the previous Section, here we shall consider the case that the possibility of the direct interaction among the particles across $\partial \mathcal{D}$ is effectively excluded, and we suppose the quasi-static limit and $V, N \rightarrow \infty$. From Eqs.(2.17) and (3.17) we have the quantitative relation

$$
\langle Q\rangle+\left\langle Q^{m i g}\right\rangle=T \Delta S+\mu \Delta\langle n\rangle,
$$

and $Q^{\partial \mathcal{D}}=0$. If we assume $U(\boldsymbol{x}, a)=\tilde{U}(\boldsymbol{x}, a)$ with Eq.(2.21), we have $Q^{m i g}=U_{0} \Delta n$. Remembering the definition of $\mu$ given by Eq.(3.10), we have,

$$
\left\langle Q^{m i g}\right\rangle \neq \mu \Delta\langle n\rangle \text {. }
$$

This leads to our main conclusion of the present Paper that each particle does not carry $\mu$ across the boundary of the system, when it enters or leaves the system.

We should notice the difference between the energy balance relation associated with the left hand side of Eq.(4.1) and the thermodynamic relation associated with the right hand side of Eq.(4.1). The thermodynamic relation is a closed expression in terms of the variation in the thermodynamic state variables of a system. On the other hand, the energy balance relation is the relation of energy exchange with the surroundings. Let us study how $\langle Q\rangle$ is expressed by using the thermodynamic state variables. From Eq.(4.1) and $Q^{m i g}=U_{0} \Delta n$, we have

$$
\langle Q\rangle=T \Delta S+k_{B} T \log \left(\rho v_{0}\right) \Delta\langle n\rangle \neq T \Delta S .
$$

This quantitative relation shows that the ensemble average of the energy transfer from the heat bath is expressed by the variation in the two thermodynamic state variables $\Delta S$ and $\Delta\langle n\rangle$ in both the two terms $T \Delta S$ and $\mu \Delta\langle n\rangle$ of the thermodynamic relation.

We should also mention the difference of the level of 
the description between the energy balance relation and the thermodynamics relation. Since the energy balance relation holds on a particular trajectory, it is not necessary for obtaining the relation to know the probability of the realization of the trajectory. In this sense, the energy balance relation describes the microscopic scale. On the other hand, in order to obtain the thermodynamic relation, it is necessary to know the probability distribution of the physical states. The thermodynamic relation is obtained so that it is a relation among the ensemble averages. In this sense, the thermodynamic relation describes the macroscopic level. Consequently, the chemical potential is regarded as the concept in the macroscopic description.

Thus, when we observe a particular process, i.e. a sample path, we can not find the chemical potential as well as the thermodynamic relation, but measure a particular value of $Q$ obeying the energy balance relation. With the increase of the number of the samples, many different values of $Q$ shall be recorded. Then, we know that $Q$ takes a value following some probability distribution. Eventually, we find the chemical potential and the thermodynamic relation by examining the ensemble of the processes.

It may be a future problem to study an energy conversion cycle of a micro-machine with at least two particle reservoirs. For example, suppose that the two reservoirs consist of the particles of the same species and provide the same one-body energy potential and the same temperature environment. Then the difference of the chemical potentials $\mu_{H}$ and $\mu_{L}$ of these reservoirs can arise only through the difference of the density of the particles. Thermodynamics tells that the maximally available mechanical work obtained per particle transported is $\mu_{H}-\mu_{L}(>0) .{ }^{4,11)}$ By studying this process with the micro-machine, it might be more evident how the small fluctuating system feels the chemical potential so as to produce the mechanical work.

1) Biological processes of biopolymers should be approached from the viewpoint of the energetics of open systems. The theoretical studies have been appeared in Refs.3, 4, where, however, the energetics of a single event of particle exchange has been discussed little.

2) A kinetic model of open systems for the description of irreversible process has been studied in the literature: J. L. Lebowitz and P. G. Bergmann, Ann. Phys. 1, 1 (1957).

3) For the purpose to characterize mechanochemical processes in biological systems, a kinetic formalism has been introduced in the literature: T. L. Hill, Prog. Biophys. Mol. Biol. 28, 267 (1974).

4) The extensive studies of Brownian motors or thermal ratchets, motivated partly by the biological motor proteins, have been appeared in the literatures: F. Jülicher, A. Ajdari and J. Prost, Rev. Mod. Phys. 69, 1269 (1997), and references cited therein. While they have implicitly included the process that particles enter or leave a system, the study of a Brownian motor taking explicit account of such a process has been appeared in Ref.10.

5) K. Sekimoto, J. Phys. Soc. Jpn. 66, 1234 (1997),

6) K. Sekimoto and S. Sasa, J. Phys. Soc. Jpn. 66, 3326 (1997)

7) K. Sekimoto, Progr. Theor. Phys. Suppl. 130, 17 (1998).

8) C. W. Gardiner, Handbook of Stochastic Methods, SpringerVerlag, (1998).
9) We may consider a dilute distribution of hard-core particles in the surroundings $\left(\mathcal{D}_{0} \backslash \mathcal{D}\right)$.

10) S. Sasa and T. Shibata, J. Phys. Soc. Jpn. 67, 1918 (1998).

11) T. Shibata and S. Sasa, J. Phys. Soc. Jpn. 67, 2666 (1998) 